\title{
Towards Low Cost Soil Sensing Using Wi-Fi
}

\author{
Jian Ding \\ Rice University
}

\author{
Ranveer Chandra \\ Microsoft Corporation
}

\begin{abstract}
A farm's soil moisture and soil electrical conductivity (EC) readings are extremely valuable for a farmer. They can help her reduce water use and improve productivity. However, the high cost of commercial soil moisture sensors and the inaccuracy of sub-1000 dollar EC sensors have limited their adoption. In this paper, we present the design and implementation of a system, called Strobe, that senses soil moisture and soil EC using RF propagation in existing Wi-Fi bands. Strobe overcomes the key challenge of limited bandwidth availability in the $2.4 \mathrm{GHz}$ unlicensed spectrum using a novel multi-antenna technique. It maps the propagation time and amplitude of Wi-Fi signals received by different antennas to the soil permittivity and EC, which in turn depend on soil moisture and salinity. Our experiments with USRP, WARP, and commodity Wi-Fi cards show that Strobe can accurately estimate soil moisture and EC using Wi-Fi, thereby showing the potential of a future in which a farmer can sense soil in their farm without investing 1000s of dollars in soil sensing equipments.
\end{abstract}

\section{CCS CONCEPTS}

- Networks $\rightarrow$ Wireless access points, base stations and infrastructure; $\bullet$ Hardware $\rightarrow$ Sensor applications and deployments; Wireless devices.

\section{KEYWORDS}

Soil sensing; Agriculture; Wi-Fi; Moisture; EC; Relative ToF; IoT

\section{ACM Reference Format:}

Jian Ding and Ranveer Chandra. 2019. Towards Low Cost Soil Sensing Using Wi-Fi. In The 25th Annual International Conference on Mobile Computing and Networking (MobiCom '19), October 2125, 2019, Los Cabos, Mexico. ACM, New York, NY, USA, 16 pages. https://doi.org/10.1145/3300061.3345440

Permission to make digital or hard copies of all or part of this work for personal or classroom use is granted without fee provided that copies are not made or distributed for profit or commercial advantage and that copies bear this notice and the full citation on the first page. Copyrights for components of this work owned by others than ACM must be honored. Abstracting with credit is permitted. To copy otherwise, or republish, to post on servers or to redistribute to lists, requires prior specific permission and/or a fee. Request permissions from permissions@acm.org.

MobiCom '19, October 21-25, 2019, Los Cabos, Mexico

(c) 2019 Association for Computing Machinery.

ACM ISBN 978-1-4503-6169-9/19/10 ..\$15.00

https://doi.org/10.1145/3300061.3345440

\section{INTRODUCTION}

Several agricultural applications rely on soil moisture and soil EC measurements. For example, precision irrigation, which refers to the variable application of water in different regions of the farm, depends on accurate soil moisture values at different depths. This technique helps reduce water use, and also reduces soil leaching and contamination of ground water by chemicals in fertilizers and other agricultural inputs. Soil EC is another key indicator of soil health. It has been shown to correlate very well with crop yield and plant nutrient availability, and farmers are recommended by the USDA to measure soil EC to determine soil treatment plans and management zones for Precision Agriculture [1].

Several techniques have been invented over the last few decades to measure soil moisture and EC. These methods include direct sensing techniques, which require soil to be extracted and dried out, as well as indirect sensing methods that measure surrogate properties of soil moisture and EC, such as capacitance, electrical, and nuclear response. Researchers have also explored the use of radar based technologies to measure soil moisture and EC.

However, one of the key challenges in the adoption of soil moisture and EC sensing technologies is the cost and accuracy of existing sensor solutions. Although hobbyist soil moisture sensors are available for less than 10 dollars, they are not reliable and degrade quickly, and are consequently not used by agricultural experts [2]. The lowest cost, commercial grade, soil moisture sensing solutions still cost over a 100 dollars each. They use ruggedized components that typically measure the resistance, capacitance, or conductivity change of the sensor (discussed in Section 2). Furthermore, most sensors measure an apparent EC, which needs to be combined with the estimated permittivity to produce an interpretable result, i.e., saturation extract EC - a measure of soil salinity. Therefore, estimating the actual salinity or saturation extract EC requires accurately measuring both the apparent EC and permittivity. We are not aware of any low cost soil sensor that can accurately estimate the salinity. Even moderately expensive (sub-1000 USD) sensors can fail to accurately estimate either apparent EC or permittivity $[3,4]$.

The high cost of soil sensors has limited the adoption of soil sensing technologies. Sensors that cost a few hundred dollars are unaffordable for most farmers in developing regions. Even in the developed world, the cost of sensors has limited the adoption of precision irrigation technologies [5]. 
In this paper we present a low-cost soil sensing technique called Strobe, for Soil Testing using RF Probes, that estimates soil moisture and soil EC without the need for a specialized sensor. Instead, Strobe leverages the phenomenon that $R F$ waves travel slower in soil with higher permittivity. Strobe uses Wi-Fi devices in the unlicensed $2.4 \mathrm{GHz}$ spectrum. With just a few antennas in soil, Strobe can estimate the permittivity and EC, and the corresponding moisture and salinity levels of soil at the location of the antennas. A wireless transmitter, e.g. a Wi-Fi card, from the soil surveying device, emits signals that are received by the antennas in soil. The receiver uses signals on multiple antennas to compute soil permittivity. The results are then transmitted back to the soil surveying device, which then computes the soil moisture and soil EC values.

Prior work on Ground Penetrating Radars (GPR) and Time Domain Reflectometery (TDR) have considered the use of RF for measuring soil properties. However, these systems are specialized, wideband, and hence cost several 1000s of dollars. They use time of flight (ToF) to measure the speed of the RF signal, and consequently the permittivity of soil. They require a wide contiguous bandwidth from $100 \mathrm{~s}$ of $\mathrm{MHz}$ to few $\mathrm{GHz}$, in the UHF spectrum, to accurately measure the ToF. However, such a wide bandwidth is not available in the UHF unlicensed spectrum. Furthermore, ToF measures the average moisture level from the surface of soil, but doesn't measure the absolute moisture levels, e.g. the soil moisture value at 8 inches below surface level. These systems rely on signal attenuation to estimate EC. Since the attenuation is affected by all parameters along the signal transmission path, the EC measurement can be error-prone.

Strobe addresses the above challenges by proposing two new techniques to estimate the moisture and EC from Wi-Fi signals. Instead of measuring the absolute ToF, which would require a wide bandwidth, Strobe measures the relative ToF of received signals between multiple antennas, which only exploits the $70 \mathrm{MHz}$ of available spectrum in $2.4 \mathrm{GHz}$. The relative ToF is used to determine permittivity and soil moisture. Since the accuracy of permittivity estimation increases over frequency, Strobe can report much more accurate soil moisture than most existing moisture sensors that use sub$100 \mathrm{MHz}$ spectrum. We then propose a new technique to measure soil EC using the ratio of signal amplitudes on the different antennas. This significantly reduces the complexity of EC estimation compared to prior RF-based methods.

To the best of our knowledge, Strobe is the first work to demonstrate the capability to sense soil moisture and soil saturation extract EC using Wi-Fi transmissions in unlicensed spectrum. This capability enables many new scenarios. For example, an EC map can help a farmer build management zones. A sprinkler system can dynamically learn moisture maps of the farm, and adapt the time of irrigation, and the amount of water that it uses in different regions.

We have implemented Strobe in the $2.4 \mathrm{GHz}$ Wi-Fi bands over various hardware, including USRP, WARP, and Qualcomm Atheros based Wi-Fi cards, and shown the system to perform as well as the more expensive soil sensors.

\section{BACKGROUND}

We first provide background on the state of the art in soil moisture and EC sensing, and then show how ToF-based techniques have used RF for soil sensing.

\subsection{Sensing soil moisture and EC}

The most accurate method for soil sensing is the direct gravimetric method [6]: of sampling soil, drying it out, and weighing the amount of moisture that is lost from the soil. However, this technique is expensive, manual, requires oven drying, and disturbs the soil.

Several lower-cost surrogate sensing approaches have been proposed in the literature that estimate soil moisture based on the indirect soil properties that are sensitive to moisture. For example, electrical resistance based sensors measure the resistance of soil when current is passed through two electrodes [7]. Capacitive sensors measure the time to charge the capacitor. A calibration equation is then used to convert the resistance to the corresponding soil moisture value. Heat-diffusion sensors exploit the fact that wet soil dissipates heat much faster than dry soil to measure the rate of temperature increase when applying a heat source [8]. Tensiometers [9] measure the tension created by soil absorbing the water kept in a ceramic cup connected through a tube. Radioactive sensors [10] measure the slowing of neutrons in soil after being emitted into the soil from a fast-neutron source. Most "commercial" grade soil moisture sensors, such as the ones from Decagon, Campbell Scientific, or Sensoterra, typically cost over a 100 dollars.

To measure EC, the resistance to current is measured through electrodes in soil. The most inexpensive sensors we are aware of cost over a 100 dollars. They have to be to connected to a microprocessor and RF modules, and hence are even more expensive.

\subsection{Soil sensing using $\mathrm{RF}$}

RF-based soil sensing is enabled by the phenomenon that $R F$ waves propagate slower and attenuate more in soil than in air due to soil's larger permittivity and EC compared to air. Figure 1 shows an overview of how the existing ToF-based RF sensing techniques, such as GPR and TDR, derive soil properties from RF wave properties. These techniques first measure electromagnetic (EM) wave velocity and attenuation in soil to infer soil apparent permittivity and apparent EC. With 


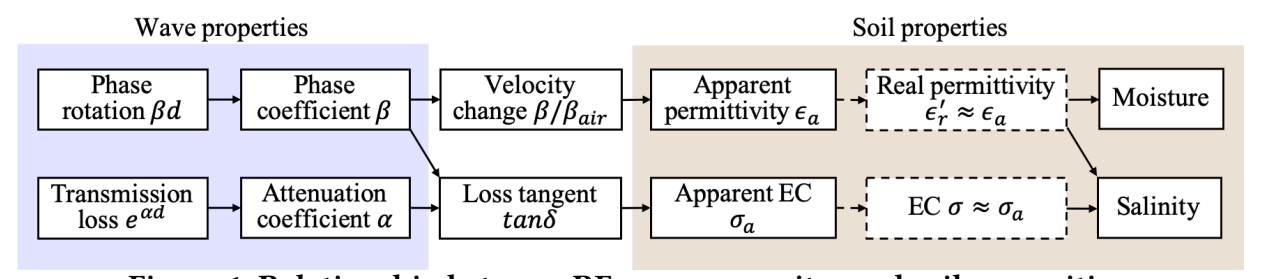

Figure 1: Relationship between RF wave properites and soil properities.

apparent permittivity and EC estimation, soil moisture and salinity can be computed from the well-studied models [1114]. Next, we introduce the soil properties shown in Figure 1 and then explain the relationship between wave propagation and soil properties.

Soil properties. In soil studies, a property, e.g., permittivity, can have various names, e.g., relative permittivity, apparent permittivity, etc., when measured in different ways. Without explaining them clearly, the readers might get confused and misunderstand the results. Therefore, here we explain the terms adopted in this paper, which are also widely used in RF sensing techniques. Within these terms, we use moisture and salinity as the end results to intuitively describe how much water and salt are contained in soil, and the other terms as ways to explain how RF sensing works and to understand Strobe's performance.

(i) Permittivity and electrical conductivity (EC) are two fundamental electrical properties of a material. Permittivity is a complex value given as $\epsilon^{*}=\epsilon^{\prime}+j \epsilon^{\prime \prime}$. EC is always considered as a real value $\sigma$ since its imaginary component is insignificant at radio frequencies [15]. The real part of permittivity $\left(\epsilon^{\prime}\right)$ dominates wave velocity, while EC $(\sigma)$ and the imaginary part of permittivity $\left(\epsilon^{\prime \prime}\right)$ dominate energy loss.

(ii) Relative permittivity (unitless) is the ratio of the absolute permittivity to the free space permittivity $\epsilon_{0}(8.854 \times$ $\left.10^{-12} \mathrm{~F} / \mathrm{m}\right)$, given as $\epsilon_{r}^{*}=\epsilon^{*} / \epsilon_{0}=\epsilon_{r}^{\prime}+j \epsilon_{r}^{\prime \prime}$. As shown in Figure 1, the real part of relative permittivity $\left(\epsilon_{r}^{\prime}\right)$ is directly related to soil moisture. For simplicity, we use the term permittivity to refer to the relative permittivity in the rest of this paper.

(iii) Apparent permittivity is the soil permittivity measured in situ. Besides the real part of permittivity $\left(\epsilon_{r}^{\prime}\right)$, apparent permittivity also captures the effects of the imaginary part of permittivity $\left(\epsilon_{r}^{\prime \prime}\right)$ and $\mathrm{EC}(\sigma)$ on wave velocity, given as:

$$
\epsilon_{a}=\frac{\epsilon_{r}^{\prime}}{2}\left[\sqrt{1+\tan ^{2} \delta}+1\right]
$$

where the loss tangent $\tan \delta$ is a measure of how lossy a soil medium is. It is a function of the complex permittivity $\left(\epsilon_{r}^{*}\right)$, EC $(\sigma)$, and measurement frequency $(f)$ :

$$
\tan \delta=\frac{\epsilon_{r}^{\prime \prime}+\frac{\sigma}{2 \pi f \epsilon_{0}}}{\epsilon_{r}^{\prime}}
$$

Existing dielectric soil moisture sensors use apparent permittivity to approximate the real part of permittivity (i.e., $\epsilon_{a} \approx \epsilon_{r}^{\prime}$ ) and estimate soil moisture, which is only accurate when $\tan \delta$ is small, e.g., with a small $\epsilon_{r}^{\prime \prime}$ and a big $f$.

(iv) Apparent EC is the EC of soil measured in situ. Its value depends on the actual $\mathrm{EC} \sigma$, the imaginary component of permittivity $\epsilon_{r}^{\prime \prime}$, and the measurement frequency $f$ :

$$
\sigma_{a}=\sigma+2 \pi f \epsilon_{0} \epsilon_{r}^{\prime \prime}
$$

Existing soil EC sensors use apparent EC to approximate the actual EC (i.e., $\sigma_{a} \approx \sigma$ ). Eq. 3 indicates that sensors operating at a very low frequency, e.g., most resistive EC sensors, can provide accurate EC estimation.

$(v)$ We use moisture to refer to the volumetric water content (VWC) $\theta$ in soil, which is the ratio of water volume to the total volume of wet soil that consists of water, air and soil particles. The models to convert $\epsilon_{a}$ to $\theta$ are soil-specific and can be found in sensor manuals, e.g., [16].

(vi) We use salinity to refer to the saturation extract EC $\sigma_{e}$ measured in siemens per meter $(\mathrm{S} / \mathrm{m}) . \sigma_{e}$ is a direct measure of how much salt is contained in soil, which can be determined from apparent permittivity and EC [16]:

$$
\sigma_{e}=\frac{\epsilon_{w} \sigma_{a}}{\epsilon_{a}-\epsilon_{\sigma_{a}=0}} \frac{\theta}{\theta_{s}}
$$

where $\epsilon_{w}$ is a the real part of permittivity of water, $\theta_{s}$ is the moisture of saturated soil, and $\epsilon_{\sigma_{a}=0}$ is the real part of permittivity when $\sigma_{a}=0$.

Wave propagation in soil. Wave propagation in soil through a distance of $d$ at frequency $f$ is modeled as:

$$
E(f, d)=A e^{-(\alpha+j \beta) d}
$$

where $\alpha$ is the attenuation coefficient that determines signal attenuation introduced by soil, $\beta$ is the phase coefficient that determines phase variation during propagation, and $A$ is the signal amplitude. $A$ is determined by antenna beam patterns, distance $d$, and system parameters such as gain settings at the transmitter/receiver and antenna gains. $\alpha$ and $\beta$ are both functions of permittivity and $\mathrm{EC}$, given as:

$$
\alpha=\frac{2 \pi f}{c} \sqrt{\frac{\epsilon_{r}^{\prime}}{2}\left[\sqrt{1+\tan ^{2} \delta}-1\right]}
$$




$$
\beta=\frac{2 \pi f}{c} \sqrt{\frac{\epsilon_{r}^{\prime}}{2}\left[\sqrt{1+\tan ^{2} \delta}+1\right]}
$$

where $c=3 \times 10^{8} \mathrm{~m} / \mathrm{s}$ is the speed of light.

As shown in Figure 1, the key to derive soil moisture and salinity from RF wave properties is to find apparent permittivity and apparent EC based on phase and amplitude changes of RF wave in soil. Next we discuss how this is done in existing RF sensing techniques.

Apparent permittivity estimation from velocity. With Eq. 1, Eq. 7 can be written as $\beta=2 \pi f \sqrt{\epsilon_{a}} / c=2 \pi f / v$, where $v=c / \sqrt{\epsilon_{a}}$ is the wave velocity in soil. Measuring wave velocity $v$ is threfore the key to estimate the apparent permtitivity $\epsilon_{a}$ of soil. ToF-based RF techniques measure the time $\tau$ it takes to travel through a known distance $d$ in soil, which gives an estimate of wave velocity as $v=d / \tau$. The apparent permtitivity $\epsilon_{a}$ can then be calculated as:

$$
\epsilon_{a}=\left(\frac{c \tau}{d}\right)^{2}
$$

Apparent EC estimation from transmission loss. RF techniques measure the signal transmission loss $e^{\alpha d}$ of wave traveling through a known distance $d$ to estimate the attenuation coefficient $\alpha$. Using $\alpha$ together with $\epsilon_{a}$ estimated from ToF measurement, the apparent $\mathrm{EC} \sigma_{a}$ can then be computed from Eq. 3, Eq. 6 and Eq. 7.

\subsection{Limitations of RF sensing techniques}

Accurate ToF and signal attenuation measurements are the key factors to get accurate moisture and EC estimation, which impose a need for specialized hardware design to give reliable results. Therefore, $\mathrm{RF}$ sensing systems is usually very expensive, on the order of several thousand dollars.

ToF sensing systems require ultra-wide bandwidth to obtain good performance, e.g., the bandwidth of systems like GPRs usually spans multiple GHz. These systems typically require specially designed hardware to allow operating on a ultra-wide frequency range and high power efficiency considering the stringent FCC-imposed power limit for ultrawideband systems, which is $-41.3 \mathrm{dBm} / \mathrm{MHz}$.

RF-based EC sensing systems have complicated design choices and calibration requirements since they rely on absolute amplitude measurements. One needs to know system parameters both during design and in operation. For TDR systems that use transmission line to estimate permittivity and EC, tradeoff exists when choosing probe design parameters for ToF and EC [17]. In antenna-based systems like GPRs, system parameters, e.g., gain settings, together with the whole propagation path from transmitter to receiver, which includes multiple reflections and refractions, need to be carefully modeled.

\section{STROBE DESIGN}

Strobe measures soil moisture and EC/salinity only using Wi-Fi signals. A Wi-Fi transmitter, such as a phone or a transmit device on a tractor, transmits packets which are received by multiple antennas in soil, as shown in Figure 2. All antennas are connected to a MIMO capable Wi-Fi device, e.g., a 3-antenna Wi-Fi card. We first use a MUSIC-based multipath resolving technique to recover the shortest path from received signal. Since the receive antennas are buried at different depths in soil, their shortest paths have different phase and amplitude changes so that we can estimate the relative ToF and amplitude among antennas, which are then used to estimate soil properties including the intermediate properties, i.e., apparent permittivity and apparent EC, and the end results, i.e., moisture and salinity. We describe these techniques in detail in the rest of this section.

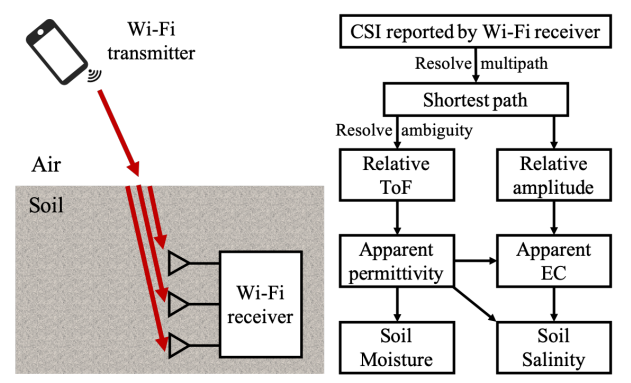

Figure 2: Overview of Strobe's hardware setup (left) and software stack (right).

\subsection{Estimating apparent permittivity}

Overcoming bandwidth limitation with relative ToF. Antennas and RF chains on a MIMO capable Wi-Fi device are synchronized in time and frequency. Previous work [18-20] has shown that such antennas can be utilized to estimate angle of arrival (AoA) based on path difference across antennas on an array. In air, this path difference, $\Delta l$, corresponds to a relative ToF of $\Delta \tau_{\text {air }}=\Delta l / c$. Our insight here is: if the path difference happens in soil, this relative ToF can be exploited to estimate soil permittivity. Since wave velocity is $\sqrt{\epsilon_{a}}$ times slower in soil than in air, the relative ToF in soil is given as $\Delta \tau_{\text {soil }}=\sqrt{\epsilon_{a}} \Delta \tau_{\text {air }}$.

In contrast to traditional absolute ToF based techniques which require ultra-wide bandwidth to achieve good accuracy, relative ToF can provide high accuracy of permittivity estimation without using wide bandwidth. The reason is that the resolution of relative ToF is constrained by carrier frequency, not bandwidth. The high accuracy of relative ToF has been demonstrated in prior AoA studies on commodity Wi-Fi devices $[18,21]$. They show that a less than 5-degree median AoA error can be achieved for a Wi-Fi device with 
3 antennas and $40 \mathrm{MHz}$ bandwidth, which can be directly mapped to a relative ToF error of $0.006 \mathrm{~ns}$ at $2.4 \mathrm{GHz}$.

Mapping relative ToF to soil permittivity. Strobe places multiple antennas in soil to create the dependency of relative ToF on soil permittivity. Typically, we are interested in a scenario where the transmitter is in air and the receiver antenna array is in soil. Since commodity Wi-Fi devices usually have three antennas, we consider using three antennas for the receive array. Next we will show how to estimate permittivity based on relative ToF estimation in this setup.

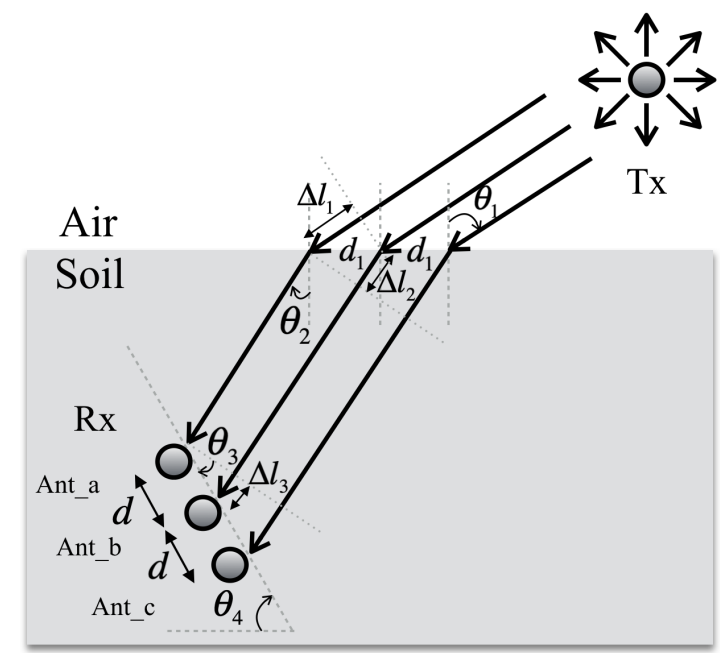

Figure 3: Model of plane wave propagating through air-tosoil surface. Transmit and receive antennas are oriented perpendicular to the plane of the paper. The wave that travels to antenna $\mathbf{B}$ has a delay of $n \Delta l_{2} / c+n \Delta l_{3} / c-\Delta l_{1} / c$ relative to the wave travels to antenna $A$.

We use an air-to-soil wave propagation model as shown in Figure 3 to derive the relationship between relative ToF and path difference in soil. For simplicity, we use the term, refractive index $n$, to describe the slow down effect of soil, which relates to permittivity as follows:

$$
n=\sqrt{\epsilon_{a}}
$$

When a signal travels from the transmitter to the receive antennas, it is refracted at the air-to-soil surface. The path difference of two adjacent antennas now has three components: $\Delta l_{1}, \Delta l_{2}, \Delta l_{3} . \Delta l_{1}$ happens in air and corresponds to a speed of $c$, while $\Delta l_{2}$ and $\Delta l_{3}$ happen in soil and correspond to a speed of $c / n$. Thus, the relative ToF of two adjacent antennas is:

$$
\Delta \tau=\frac{\Delta l_{1}}{c}-\frac{n \Delta l_{2}}{c}+\frac{n \Delta l_{3}}{c}=\frac{\Delta l}{c}
$$

where $\Delta l=\Delta l_{1}-n \Delta l_{2}+n \Delta l_{3}$ is the effective total path difference. Next, we rely on geometry and Snell's law to find out the relationship between $\Delta l$ and $n$. First, we compute $\Delta l_{1}$, $\Delta l_{2}$ and $\Delta l_{3}$ as follows:

$$
\Delta l_{1}=d_{1} \sin \theta_{1}, \Delta l_{2}=d_{1} \sin \theta_{2}, \Delta l_{3}=d \sin \theta_{3}
$$

where $d$ is the distance between antennas on the antenna array, $d_{1}$ is the distance between waves going to the antenna array at the air-to-soil surface, $\theta_{1}$ is the angle of incidence, $\theta_{2}$ is the angle of refraction, and $\theta_{3}$ is the angle of incidence at the antenna array.

Since the refraction at air-to-soil surface follows Snell's law, $\theta_{1}$ relates to $\theta_{2}$ as $\sin \theta_{1}=n \sin \theta_{2}$. Therefore, we have $\Delta l_{1}=n \Delta l_{2}$ and $\Delta l$ is simplified to $\Delta l=n d \sin \theta_{3} . \theta_{3}$ is determined by the angle of refraction $\theta_{2}$ and the angle of antenna array's rotation $\theta_{4}$, given as $\theta_{3}=\theta_{4}-\theta_{2}$. We can then rewrite $\Delta l$ as a function of array parameters, i.e., $d$ and $\theta_{4}$, angle of incidence $\theta_{1}$, and refractive index $n$ :

$$
\Delta l=n d \sin \left(\theta_{4}-\theta_{2}\right)=n d \sin \left(\theta_{4}-\arcsin \left(\frac{\sin \theta_{1}}{n}\right)\right)
$$

From Eq. 9, Eq. 10 and Eq. 12, we can compute $\epsilon_{a}$ from the relative ToF $\Delta \tau=\Delta l / c$ if we know $d, \theta_{4}$ and $\theta_{1}$, which is possible because $d, \theta_{4}$ and $\theta_{1}$ are all independent of soil moisture and we can control them to be constants during the deployment of the receive antenna array and the transmit antenna. Note that in the case of normal incidence, $\theta_{1}$ is 0 and Eq. 12 can be simplified to $\Delta l=n d \sin \theta_{4}$.

\subsection{Estimating apparent EC}

As discussed in Section 2, measuring apparent EC from absolute signal attenuation is prone to errors, and is difficult to implement and calibrate. Instead, we propose a new technique that uses the ratio of amplitudes across multiple antennas, which we call relative attenuation, to estimate EC. This eliminates the need to calibrate several system parameters, such as antenna gains and impedance.

Absolute attenuation model. To explain the advantage of using relative attenuation, we first look at the absolute attenuation between omni-directional transmit and receive antennas during air-to-soil transmission [22]:

$$
\frac{P_{t}}{P_{r}}=\underbrace{T}_{\text {refraction }} \underbrace{\frac{1}{G_{t} G_{r}}}_{\text {antenna gains }} \underbrace{\left(\frac{4 \pi\left(d_{s} \sqrt{\epsilon_{a}}+d_{a}\right) f}{c}\right)^{2}}_{\text {spreading loss }} \underbrace{e^{2 \alpha d_{s}}}_{\text {transmission loss }}
$$

where $d_{s}$ and $d_{a}$ are the distances that the wave travels in soil and air. $T$ is a transmission coefficient due to refraction at air-to-soil interface, which is a function of wave incident angle and soil permittivity. To get the absolute attenuation, a system needs to measure all the parameters along the airto-soil transmission path as shown in Eq. 13. 
Reducing model complexity with relative attenuation. In Strobe, the multiple receive antennas allow us to simplify EC estimation with relative attenuation by leveraging an insight: three closely-located and orientation-aligned antennas experience similar signal attenuation along the transmission path except the path differences among antennas. With this insight, we can eliminate the need to measure the transmission coefficient $T$ and antenna gains, $G_{r}$ and $G_{t}$, for the computation of relative attenuation, thus making Strobe less error-prone in practice. To derive the model for relative attenuation, we can assume a same $T$ for the three receive antennas because they have similar transmission paths. Furthermore, since soil moisture does not vary much within a small area, the three antennas experience a similar impedance change and hence can be assumed to have the same antenna gain $G_{r}$. The receive antennas simultaneously receive the same packet from the same transmitter and thus have the same $G_{t}$. The model of relative attenuation between two adjacent receive antennas is then given as:

$$
\frac{P_{r}\left(d_{s_{1}}, d_{a_{1}}\right)}{P_{r}\left(d_{s_{2}}, d_{a_{2}}\right)}=\left(\frac{d_{s_{2}} \sqrt{\epsilon_{a}}+d_{a_{2}}}{d_{s_{1}} \sqrt{\epsilon_{a}}+d_{a_{1}}}\right)^{2} e^{2 \alpha\left(d_{s_{2}}-d_{s_{1}}\right)}
$$

In the case of normal incident $\left(d_{a_{1}}=d_{a_{2}}\right)$ and far field, the above equation can be reduced to $P_{r e l}(\Delta d)=e^{2 \alpha \Delta d}$.

\subsection{Soil-specific design choices}

As indicated by Eq.12 and Eq.14, the choice of antenna array parameters play an important role in Strobe's performance. Specifically, these parameters are: $(i)$ antenna array rotation $\theta_{4}$ and ( $i$ ) antenna distance $d$. In addition, we need to choose a proper frequency band as the wave's carrier frequency.
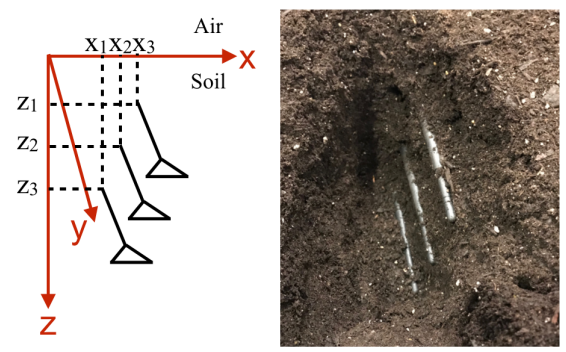

Figure 4: A typical antenna array setup in soil. The antennas are buried at different depths and the distance between two adjacent antennas in the horizontal plane is small.

3.3.1 Choices of array parameters. We make our choices of antenna distance $d$ and antenna array rotation $\theta_{4}$ to reduce the effects of soil surface roughness and soil non-homogeneity. Figure 4 shows a real-world example of an antenna setup in soil. We choose a small antenna distance in the $x$-axis and a relatively big antenna distance in the $z$-axis, i.e., a $\theta_{4}$ close to 90 degrees and a relatively big $d$. Next we explain how such choices are derived.

All the equations in Section 3.1 are based on the assumption that the soil surface is totally flat and soil is a homogeneous medium. However, in practice soil surface is always rough and soil moisture can vary a lot when measured in a small volume while it is stable when averaged over a large volume. The roughness of soil surface and non-homogeneity of soil medium, if not taken care of when choosing array parameters, can make Eq.12 and Eq.14 inaccurate.

To reduce the impact of soil surface roughness, we should constrain the incident points of waves going to different antennas to be within a small area to make sure the assumption that the waves have similar paths holds. In the ideal case, this leads to $\theta_{4}=90^{\circ}$. However, when $\theta_{4}=90^{\circ}$, i.e., the antennas are vertically aligned, the top antenna is likely to block the line-of-sight (LoS) paths of the bottom two antennas. Therefore, we choose $\theta_{4}$ to be a value around 90 degrees that does not cause blockage.

Since the average soil moisture is more stable when averaged over a larger volume, we should use a big $d$ to reduce the impact of soil non-homogeneity. However, it is also problematic when $d$ is too big due to the fast signal attenuation in soil as well as an ambiguity issue that we will discuss in Section 3.5. Hence, $d$ should fall in a range that is neither too small nor too big. In practice, we determine the value of $d$ experimentally (Section 5.1.2).

3.3.2 Frequency band selection. Eq. 13 indicates that signal attenuation in soil is frequency-dependent. Higher frequency signals have higher attenuation. In Strobe, we should choose a relatively low frequency that at least allows the wave to propagate to the bottom antenna.

To understand how Wi-Fi frequency bands, i.e., $2.4 \mathrm{GHz}$ and $5 \mathrm{GHz}$., attenuate in soil at different moisture levels, we conduct measurements with a vector network analyzer (VNA) in potting soil. With a transmission power of $15 \mathrm{dBm}$, the VNA is not able to provide useful information when the $\log$ magnitude is smaller than $-90 \mathrm{~dB}$. Figure 5 plots signal attenuation in soil for the three receive antennas at depths of $5 \mathrm{~cm}, 10 \mathrm{~cm}$ and $15 \mathrm{~cm}$ in soil. We can see that the $2.4 \mathrm{GHz}$ spectrum maintains larger than $-80 \mathrm{~dB} \log$ magnitudes at all moisture levels while the $5 \mathrm{GHz}$ spectrum does not have a high-enough signal strength for the bottom antenna even when soil is very dry. Due to the high attenuation, the $5 \mathrm{GHz}$ spectrum is not appropriate for soil sensing, although it has a total bandwidth that spans about $665 \mathrm{MHz}$. These results indicate that we should focus on using $2.4 \mathrm{GHz}$ channels, which only have around $70 \mathrm{MHz}$ of available bandwidth. 


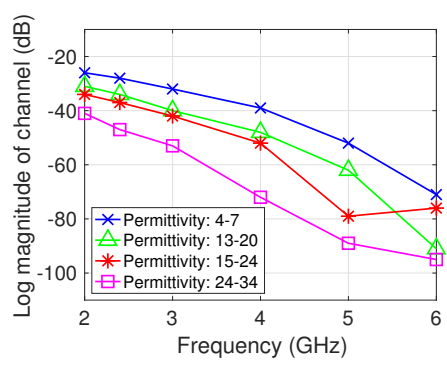

(a) Depth: $5 \mathrm{~cm}$

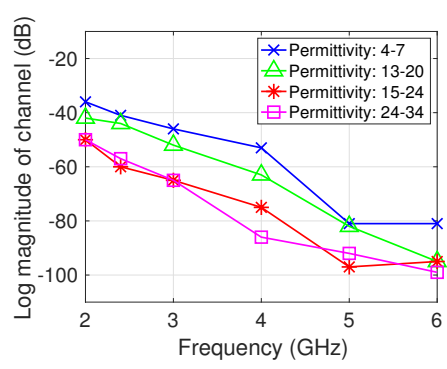

(b) Depth: $10 \mathrm{~cm}$

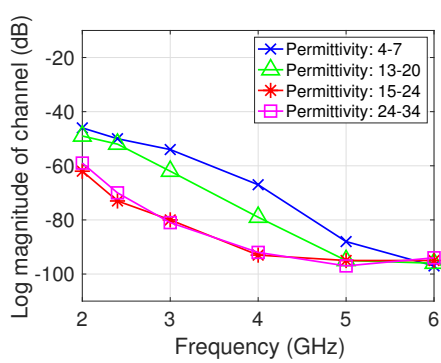

(c) Depth: $15 \mathrm{~cm}$

Figure 5: Channel attenuation in soil at different depths measured by network analyzer. Generally, signal attenuation increases as frequency, depth, or soil moisture increases.

\subsection{Resolving multipath}

The equations derived in Section 3.1 only consider the shortest path from the transmit to the receive antennas. In practice, channels always consist of multiple paths. In our measurement setup, the shortest path is also the strongest path in most cases. Therefore, we use the MUSIC algorithm to accurately recover the shortest path from a multipath channel. Another practical issue is that if there is no time and frequency synchronization between the transmitter and the receiver, the measured CSI would be corrupted by hardware impairments, e.g., packet detection delay (PDD), sampling frequency offset (SFO), and carrier frequency offset (CFO). Similar to AoA-based methods, e.g., SpotFi [18], Strobe does not require time and frequency synchronization to apply MUSIC for relative ToF estimation. Next, we mathematically explain the reason.

In a multipath environment, the CSI of the $m^{t h}$ antenna and the $n^{\text {th }}$ frequency can be written as the sum of $L$ paths:

$$
h_{m, n}=\sum_{l=1}^{L} a_{l, m} e^{-j 2 \pi\left(f_{0}+\Delta f n\right) \tau_{l, m}}
$$

where $a_{l, m}$ is a complex-valued amplitude of $l^{t h}$ path, $\tau_{l}$ is the absolute ToF of $l^{t h}$ path and $\Delta f$ is the frequency spacing between two adjacent frequency samples.

Without time and frequency synchronization between the transmitter and the receiver, the corrupted CSI is given as:

$$
\hat{h}_{m, n}=\sum_{l=1}^{L} a_{l, m} e^{-j \theta_{0}} e^{-j 2 \pi\left(f_{0}+\Delta f n\right)\left(\tau_{l, m}+\tau_{0}\right)}
$$

where $\theta_{0}$ is the phase shift caused by CFO and $\tau_{0}$ is the ToF shift caused by PDD, SFO, and other possible delays in hardware. $\theta_{0}$ and $\tau_{0}$ are the same across all the paths, subcarriers, and antennas within a single channel because these samples are measured at the same time and therefore experience the same hardware impairments. Hence, although we do not know what $\tau_{0}$ is, the relative ToF between two antennas is not affected by hardware impairments, i.e., $\Delta \tau=$ $\tau_{l, i}-\tau_{l, j}=\left(\tau_{l, i}+\tau_{0}\right)-\left(\tau_{l, j}+\tau_{0}\right)$. For a uniform linear antenna array, the path difference remains the same for all adjacent antenna pairs under far-field assumption, so does the relative ToF, i.e., $\Delta \tau_{l}=\tau_{l, i}-\tau_{l, i+1}=\tau_{l, i+1}-\tau_{l, i+2}$. Similar to SoptFi, we can then use MUSIC to jointly estimate absolute ToF $\left(\tau_{l, m}-\tau_{0}\right)$ and relative ToF $\left(\tau_{l, i}-\tau_{l, j}\right)$ from corrupted CSIs. The absolute ToF here contains PDD, SFO, and delays from hardware, and thus is discarded by Strobe. Only the relative ToF is remained and used for later apparent permittivity and EC estimation.

\subsection{Resolving ambiguity in relative ToF}

Existing over-the-air AoA methods usually adopt a halfwavelength antenna distance to avoid ambiguous results. In soil, however, ambiguity is easier to occur due to the higher permittivity (a physical distance of $d$ is equivalent to an effective distance of $n d$ ) and hard to avoid because antenna distance cannot be too small (Section 3.3.1).

We first explain how phase ambiguity leads to relative ToF ambiguity. For the three receive antennas in our setup, the phase rotations of received signals are: $\theta_{1}=-2 \pi f \tau$, $\theta_{2}=-2 \pi f(\tau+\Delta \tau)$ and $\theta_{3}=-2 \pi f(\tau+2 \Delta \tau)$, where $\tau$ is the absolute ToF, $\Delta \tau$ is the relative ToF, and $f$ is the carrier frequency. We measure the phase rotations to estimate the relative ToF $\Delta \tau$. However, the measured values are ambiguous because the actual value of a phase rotation can be all possible values of $2 \pi k+\theta$, where $k$ is an arbitrary integer and $\theta$ is the measured phase rotation falling in $[0,2 \pi)$. The relative ToF thus can have ambiguous values of $\Delta \tau, \Delta \tau+\tau_{0}$, $\Delta \tau+2 \tau_{0}$, etc., where $\tau_{0}=1 / f$ is the time for the phase to rotate $2 \pi$.

This ambiguity is also known as spatial aliasing in previous work, e.g., AWL [23]. AWL proposes a method to resolve this issue by exploiting both $2.4 \mathrm{GHz}$ and $5 \mathrm{GHz}$ bands. However, this method does not apply to Strobe because $5 \mathrm{GHz}$ signals do not propagate well through soil. Next we show how Strobe leverages the knowledge of soil properties to 
remove this ambiguity. First, we rely on the knowledge about the range of soil's refraction index $n$, which is usually between 2 and 6, to reduce the number of ambiguous values. For example, when the antenna depth difference is $4.5 \mathrm{~cm}$, the corresponding range of relative ToF is $0.3-0.9 \mathrm{~ns}$, where ambiguity only occurs when the relative ToF falls in $0.3-0.5$ ns or $0.7-0.9 \mathrm{~ns}$ for a $2.4 \mathrm{GHz}$ signal ( $\left.\tau_{0}=0.4 \mathrm{~ns}\right)$. Thus, there are at most 2 ambiguous results. From these limited number of results, we can rely on signal amplitudes to pick the correct one. In practice, the gap between the amplitudes corresponding to the ambiguous ToF ranges is big enough to make this method reliable, e.g., $20 \mathrm{~dB}$ as shown in Figure 5. We can further improve the reliability by utilizing amplitudes measured by all three antennas and at multiple transmit antenna locations to reduce the impacts of multipath and transmit antenna location change.

\subsection{Calibration for frequency-dependent soil properties}

Similar to most dielectric-based commodity soil sensors, Strobe measures apparent permittivity $\epsilon_{a}$ and EC $\sigma_{a}$ of soil, which are frequency-dependent as shown in Eq. 1 and Eq. 3. Commodity soil sensors usually use $\epsilon_{a}$ to approximate the real part of permittivity (i.e., $\epsilon_{a} \approx \epsilon_{r}^{\prime}$ ) and $\sigma_{a}$ to approximate the actual EC (i.e., $\sigma_{a} \approx \sigma$ ) to simplify their conversions to moisture and salinity, which could lead to erroneous results when the approximations do not hold [24-26]. Specifically, at lower frequencies, $\sigma$ has a significant impact on $\epsilon_{a}$ so that $\epsilon_{a}$ can largely overestimate $\epsilon_{r}^{\prime}$; at higher frequencies, $\epsilon_{r}^{\prime \prime}$ has a significant impact on $\sigma_{a}$ so that $\sigma_{a}$ can largely overestimate $\sigma$. Since Strobe operates at a high frequency, i.e., $2.4 \mathrm{GHz}$, we calibrate estimated $\sigma_{a}$ to get $\sigma$. For permittivity, Strobe is able to compute $\epsilon_{r}^{\prime}$ from $\epsilon_{a}$. We note that they are almost the same at $2.4 \mathrm{GHz}$, so we use $\epsilon_{a}$ to approximate $\epsilon_{r}^{\prime}$.

Calibration method for EC. Similar to the calibration methods adopted by existing soil sensors, we perform a linear regression to match EC estimated by Strobe with ground truth EC. The linear relationship is given as:

$$
\sigma_{c a l i}=a\left(\sigma_{\text {raw }}-b\right)
$$

The choice of the linear model is based on our experiments. We notice that such a linear relationship has been reported in prior work on TDR [26]. A possible reason for why this linear relationship holds is that both $\epsilon_{r}^{\prime \prime}$ and $\sigma$ increases as soil moisture increases.

Converting to moisture and salinity. Strobe exploits models given in a soil sensor manual [16] to convert the raw apparent permittivity $\epsilon_{a}$ and calibrated apparent EC $\sigma_{c a l i}$ to soil moisture and salinity.

\section{IMPLEMENTATION}

We implement Strobe on multiple platforms including USRP, WARP, and off-the-shelf Wi-Fi cards to measure soil moisture and EC at $2.4 \mathrm{GHz}$. USRP allows us to do wideband experiments for ground truthing. The WARP board allows us to replicate CSI measurements similar to Wi-Fi cards. Since WARP has better support for manual configurations, especially gain settings, we microbenchmark the performance of Strobe mainly with WARP. To show that Strobe can be deployed on low-cost commodity hardware, we validate our results with off-the-shelf Wi-Fi cards. We test two open source CSI tools [27, 28] with Intel Wi-Fi Link 5300 NIC and Atheros AR9590 Wi-Fi NIC. Since the Intel Wi-Fi cards have a well known issue of random phase jumps at $2.4 \mathrm{GHz}$ [20], we choose to use the Atheros cards in our experiments.

USRP setup and calibration. We take wideband measurements that span from $400 \mathrm{MHz}$ to $1400 \mathrm{MHz}$ using two USRP N200 devices with SBX daughterboards, one as transmitter and the other as receiver. We choose a much smaller frequency range than the range the boards can operate on, i.e., 400-4400 MHz, because we observe that at higher frequencies, the SBX daughterboards have very low transmission power, thus producing unreliable CSI data. To emulate a MIMO capable receiver equipped with multiple antennas as described in Section 3, we switch antennas during the measurements. For each antenna, the system sweeps through the $400-1400 \mathrm{MHz}$ spectrum with a step size of $5 \mathrm{MHz}$.

To allow such an emulation, PLL offsets, CFO, SFO, and PDD must be consistent for all the receiver antennas. We employ three methods to calibrate them: $(i)$ We exploit a PLL phase offset resync feature on the SBX daughterboards to synchronize PLL phase offsets on two USRPs after each frequency retune; (ii) We use a MIMO cable to get time and frequency synchronization of two devices. (iii) We use a narrowband sinusoid for CSI estimation to reduce PDD effect.

WARP and Wi-Fi card setup and calibration. With WARP boards and Wi-Fi cards, we take narrower bandwidth measurements that only exploit the $70 \mathrm{MHz}$ Wi-Fi spectrum at $2.4 \mathrm{GHz}$. Unlike the USRP measurements, here we consider a more practical case that transmitter and receiver are not time and frequency synchronized.

We use the WARPLab reference design [29] to implement CSI measurement on WARP boards. To emulate the Wi-Fi cards, we use the pilot sequence from the $802.11 \mathrm{n} \mathrm{Wi-Fi}$ standard to estimate CSI. One WARP board connects to three receive antennas and another connects to a single transmit antenna. In all the experiments, we use a fixed transmit power of $8 \mathrm{dBm}$, which is much lower than the FCC-imposed power limit for $2.4 \mathrm{GHz}$ channels.

For the Wi-Fi cards, we use the Atheros CSI tool [28] to collect CSI and set the cards into monitor-injector mode to 
get CSI with stable phase. We install the two Wi-Fi cards on two laptops through mini-PCIe to ExpressCard apdaters. We use one Wi-Fi card connected with three antennas as the receiver and another connected with one antenna as the transmitter.

To use the entire $70 \mathrm{MHz}$ bandwidth, we switch all the radio frequencies across the $2.4 \mathrm{GHz}$ channels. We exploit two common procedures to calibrate the inconsistent impacts of hardware impairments across channels for both WARP boards and Wi-Fi cards: (i) We use a wired connection between the transmit antenna and the three receive antennas to calibrate the PLL phase offsets. Based on our experiments ${ }^{1}$, such a calibration only needs to be performed once for all channels and there is no need to re-calibration unless radios are reset. (ii) We adopt SpotFi's phase sanitization algorithm [18] to equalize the impact of PDD and SFO on channel phase slopes across multiple channel measurements.

Data analysis framework. We implement a data analysis framework in Matlab that can analyze CSI data in realtime and display soil moisture and EC values over time. The framework can either read CSI data collected by the WARP board using WARP's Matlab APIs or import CSI data from the Atheros CSI tool by opening up a TCP connection between the CSI tool and the Matlab framework.

Antenna array. To reduce deployment efforts, we use a box to hold the antennas at correct relative positions, i.e., fixed antenna distance and array rotation, as shown in Figure 6(a). This box is made waterproof to protect the connectors of antennas. There is a rod coming out from soil surface to tell the farmers where the antennas are buried.

Experimental setup. In our experiments, only the receive antennas are buried and they are connected to either a WARP board or a Wi-Fi card installed on a laptop through SMA cables with the same length. As shown in Figure 6, we setup potting soil boxes in a tent to conduct measurements with controlled salinity and moisture levels, and test real soils in outdoor environments.

\section{PERFORMANCE EVALUATION}

We first microbenchmark Strobe's relative ToF accuracy under various settings, i.e., different receive antenna distances, bandwidths, and moisture levels, and then evaluate its performance of estimating different levels of soil permittivity/moisture and EC/salinity at $2.4 \mathrm{GHz}$ Wi-Fi spectrum with fixed antenna distance and bandwidth.

Baselines. In our microbenchmarks, we compare narrowband relative ToF adopted in Strobe against absolute ToF

\footnotetext{
${ }^{1}$ We observe that on WARP boards, PLL phase offset of a channel remains the same after frequency retune, although different channels have different offsets. For the Wi-Fi cards, tracking phase jump after frequency retune is simple because the RF chains share the same PLL and their random phase only has two possible states separated by $\pi$.
}

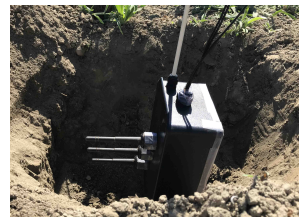

(a)

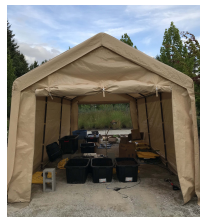

(b)

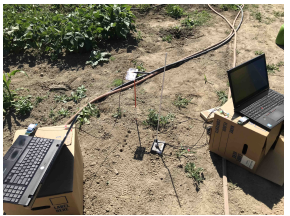

(c)
Figure 6: Soil measurement setup for multi-antenna system. Antennas are at different depths in soil while there is a rod coming out from soil surface to indicate the location of antenna array in soil. (a) Receive antenna array on a waterproof box. (b) Tent with soil boxes. (c) Measurement setup on a farm.

and wideband relative ToF. For all the soil experiments, we compare Strobe against a commodity soil sensor. More specifically, we make the following comparisons: (i) Absolute ToF vs. relative ToF: We perform this comparison in air to demonstrate the advantage of using relative ToF in achieving high accuracy. (ii) Wideband vs. narrowband: For relative ToF, we compare its accuracy when operating with different bandwidths, both in air and in soil, to show that its accuracy is not constrained by bandwidth. We use USRPs for the first two comparisons because USRPs allow flexible bandwidth settings within a $1 \mathrm{GHz}$ total bandwidth. (iii) $2.4 \mathrm{GHz}$ wideband vs. $2.4 \mathrm{GHz}$ narrowband: Since the USRPs we use do not work well at $2.4 \mathrm{GHz}$ (Section 4), we compare narrowband results measured by WARPs against wideband results measured by a VNA. (iv) Commodity sensor vs. Strobe: We compare Strobe's permittivity and EC performance against a commodity soil sensor, Decagon GS3, which can simultaneously measure apparent permittivity, apparent EC and temperature. The sensor has \pm 1 accuracy of measuring permittivity in 1-40 range and $\pm 15 \%$ accuracy in $40-80$ range. It has $\pm 10 \%$ accuracy of apparent EC measurement in the range of $0-0.5 \mathrm{~S} / \mathrm{m}$. The sensor operates at $70 \mathrm{MHz}$.

Metrics. (i) We use ToF for over-the-air evaluations. (ii) We report apparent permittivity (unitless) and EC (S/m) values, which are the default outputs from the Decagon GS3 sensor and commonly adopted by commodity soil sensors. (iii) To make the results more intuitive, we also report soil moisture (i.e., volumetric water content) and salinity (i.e., saturated extract EC), which are the metrics widely used in soil moisture and salinity studies. We apply the models given in the Decagon GS3 sensor manual [16] for both the soil sensor and Strobe to convert permittivity to moisture and apparent EC to saturated extract EC.

\subsection{Relative ToF estimation accuracy}

In this evaluation, we show that Strobe is able to accurately estimate relative ToF with a very small bandwidth. We 
first use USRPs operating over $1 \mathrm{GHz}$ bandwidth to microbenchmark, and then use WARPs to evaluate the performance at $2.4 \mathrm{GHz}$.

5.1.1 ToF accuracy over the air . Since soil is not a homogeneous medium and has permittivity/moisture variations, we first conduct over-the-air measurements to isolate ToF estimation error introduced by Strobe from the variations introduced by soil. We evaluate Strobe's relative ToF estimation accuracy with different receive antenna distances and bandwidths. To emulate moisture level increase in soil, we increase antenna distances in air to get longer relative ToFs.

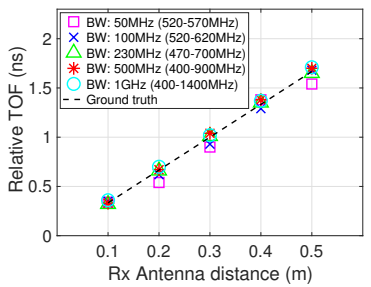

(a)

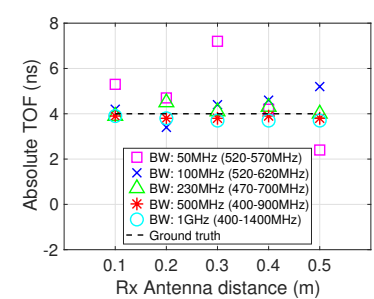

(b)
Figure 7: Strobe's joint relative and absolute ToF estimation performance over the air. (a) Relative ToF: Using 3 antennas to jointly estimate relative ToF and absolute ToF gives very accurate results even with a small bandwidth. (b) Absolute ToF of the antenna closest to the transmit antenna: Absolute ToF deviates more with smaller bandwidths.

We use USRPs to collect CSI data in an indoor environment under strong LoS conditions. We vary the distance between adjacent receive antennas from $0.1 \mathrm{~m}$ to $0.5 \mathrm{~m}$. The distance between the transmit antenna and the receive antenna closest to it is $1.2 \mathrm{~m}$ and remains the same across all the measurements. The ground truth ToF is the distance measured by a tape measure and divided by speed of light. Since the two USRP devices are time and frequency synchronized, we estimate both relative ToF and absolute ToF from collected CSI data using a joint estimation method, which estimates relative ToF and absolute ToF at the same time.

We observe from Figure 7 that the errors of relative ToF are small even with only $50 \mathrm{MHz}$ bandwidth while the errors of absolute ToF increase significantly as bandwidth reduces. Note that this absolute ToF is produced from the joint estimation method which exploits data from all three antennas to improve the accuracy of absolute ToF. If only using one antenna, the absolute ToF errors will be even larger. These results indicate that Strobe can indeed overcome the bandwidth limit that constrains the accuracy of absolute ToF. However, the slight increase of relative ToF error with smaller bandwidth also indicates that larger bandwidth can help further improve Strobe's accuracy in a multipath environment.
5.1.2 Relative ToF Accuracy in Soil . Here we first use USRPs to microbenchmark Strobe's performance of estimating relative ToF in soil with different antenna distances, bandwidths and moisture levels, and then use WARPs to examine Strobe's performance with $70 \mathrm{MHz}$ bandwidth at $2.4 \mathrm{GHz}$. We conduct the experiments in a potting soil box. We bury the receive antennas at different depths in soil and put the transmit antenna at a certain height above soil surface. We use a small horizontal distance of $2 \mathrm{~cm}$ between two adjacent antennas. The height is $1.08 \mathrm{~m}$ in USRP measurements and $0.36 \mathrm{~m}$ in WARP measurements. In each experiment, we compare Strobe's results against the Decagon GS3 soil sensor. We use the soil sensor to measure moisture at more than 10 locations in the area around the antenna array to take care of soil heterogeneity.

Impact of antenna depth difference. As discussed in 3.3.1, antenna distance is a key factor in our antenna array design that affects the relative ToF estimation accuracy. We vary the distance between receive antennas in the vertical plane in this evaluation. Figure 8(a) plots the permittivity estimated from relative ToF. We observe that the heterogeneous nature of soil affects both the sensor and Strobe. The permittivity data collected by soil sensor shows that soil moisture can vary within a certain range in an area. We observe that when using Strobe with a depth difference of 1.5 $\mathrm{cm}$, the estimated permittivity can deviate a lot from sensor data and wider bandwidth cannot help improve performance. This is because $1.5 \mathrm{~cm}$ is relatively small compared to possible path length variations caused by soil heterogeneity. With a larger depth difference, the permittivity values estimated with different bandwidths are more converged. Based on these observations, we use a depth difference of $4.5 \mathrm{~cm}$ in the following evaluations.

Relative ToF at different moisture levels. We vary soil moisture by adding tap water into soil, which has a EC value of $0.006 \mathrm{~S} / \mathrm{m}$ according to the soil sensor. We measure the accuracy of Strobe in determining different soil moisture levels. In each experiment, we stir the soil thoroughly to mix water into soil before burying the antenna array. Figure 8(b) shows results from USRPs at different moisture levels and with different bandwidths. At all moisture levels, the estimated permittivity does not deviate too much from the sensor data, even with a small bandwidth. For the highest moisture level, we observe the results of different bandwidths diverge more. This is because of the hardware impairment of SBX daughterboards discussed in 4. At a high moisture level, signal attenuates a lot so that the CSIs at higher frequencies become unreliable.

Figure 8(c) shows the estimated permittivity at $2.4 \mathrm{GHz}$ measured by WARP with a bandwidth of $70 \mathrm{MHz}$. We compare Strobe against both the soil sensor and VNA. For the VNA measurements, we only change the signal generator 


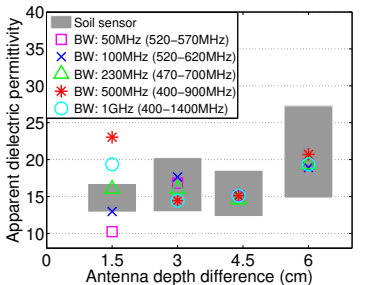

(a)

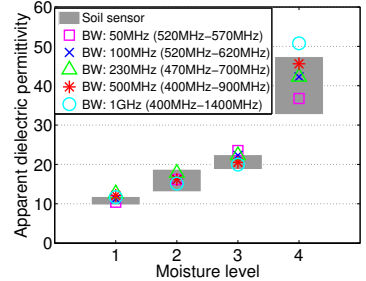

(b)

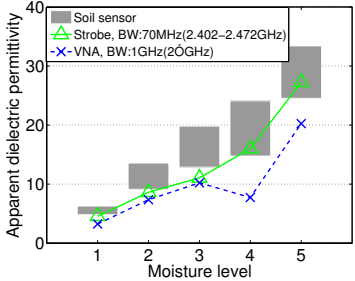

(c)

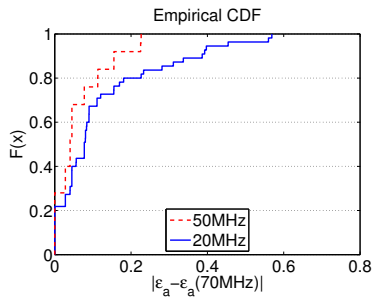

(d)

Figure 8: Soil permittivity estimation based on relative ToF. (a) USRP experiments over antenna depth differences: a small depth difference results in large estimation error. (b) USRP experiments over moisture levels: even a small bandwidth is enough to distinguish soil moisture levels. (c) $2.4 \mathrm{GHz}$ WARP experiments: permittivity estimation by WARP is more accurate compared against VNA; permittivity estimated at $2.4 \mathrm{GHz}$ is smaller than soil sensor results measured at $70 \mathrm{MHz}$. (d) $\mathrm{CDF}$ of permittivity deviations when using smaller bandwidth: using $20 \mathrm{MHz}$ can result in slightly higher variations.

and recorder from WARP to VNA while leaving the antennas at the same locations. We measure channel phase rotation over $1 \mathrm{GHz}$ bandwidth to get the absolute ToF for all the antennas and then calculate relative ToF between antennas. We observe that the soil sensor and Strobe have a consistent permittivity increase as moisture level increases. However, the VNA is only consistent with the soil sensor and Strobe under low moisture levels. Since we do not perform multipath processing for VNA data, it does perform well very under high moisture levels. We also notice that permittivity values estimated at higher frequencies by both WARP and VNA are slightly smaller than those measured by the soil sensor. This is because soil permittivity is frequency-dependent, as discussed in Section 3.6.

Relative ToF with narrower bandwidths. Here we evaluate the impact of bandwidth on permittivity estimation at $2.4 \mathrm{GHz}$. For all moisture levels shown in Figure 8(c), we compute permittivity values for $20 \mathrm{MHz}$ and $50 \mathrm{MHz}$ bandwidths by subsetting the $70 \mathrm{MHz}$ data collected by WARP. We include all possible subsets in Figure 8(d), which shows that a smaller bandwidth can result in more variations of permittivity. However, we observe that even for a $20 \mathrm{MHz}$ bandwidth, the maximum permittivity deviation is only 0.6 , which is negligible for soil moisture estimation.

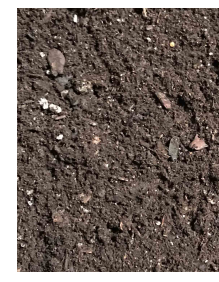

(a) Potting mix

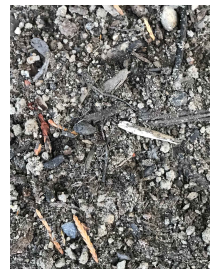

(b) Sandy loam

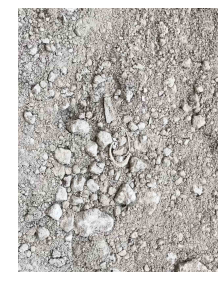

(c) Silt loam
Figure 9: Soil types used in experiments

\subsection{Permittivity and EC at Wi-Fi Spectrum}

Here we seek to answer a key question: does the permittivity and EC estimated by Strobe match the results in wellestablished soil studies under various soil conditions? We consider three major factors, soil moisture, soil salinity and soil type, that affect permittivity and EC in soil. We evaluate how Strobe acts on soil permittivity and EC changes introduced by these factors. We control them separately: (i) Moisture: We add tap water into soil to create different moisture levels. (ii) Salinity: Since controlling salinity in-situ is non-trivial, we setup three potting soil boxes with three salinity levels by adding different amounts of salt into them. (iii) We test three types of soil as shown in Figure 9: potting mix and two types of real soil - sandy loam and silt.

We conduct measurements with WARP at $2.4 \mathrm{GHz}$ and compare the results against the Decagon GS3 soil sensor. Figure 11(a) plots the raw permittivity and EC outputs from Strobe and the soil sensor, each with 5 curves. Each curve contains data at different moisture levels with a single soil type and at a single salinity level. For each data point, we average WARP results at multiple heights of the transmit antenna from $0.15 \mathrm{~m}$ to $0.6 \mathrm{~m}$ and sensor results at more than 10 locations around the antenna array.

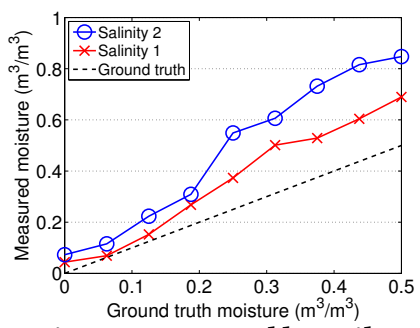

Figure 10: Soil moisture measured by soil sensor at two salinity levels. The sensor reports higher moisture values at the higher salinity level. 


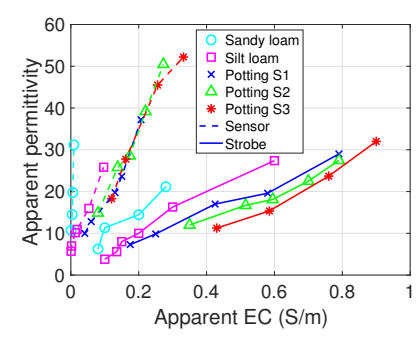

(a) Raw: EC-permittivity

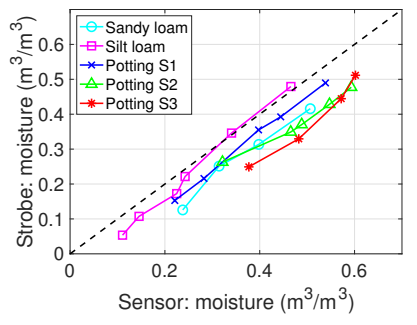

(b) Raw: moisture-moisture

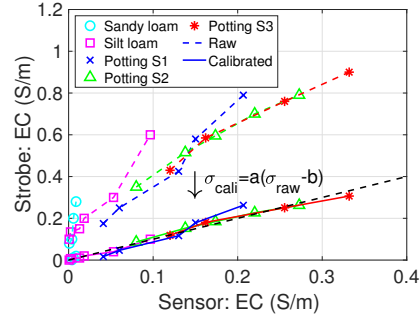

(c) Strobe calibrated

Figure 11: Soil permittivity and EC estimation with Strobe and Decagon GS3 sensor. Strobe calibration: EC is calibrated based on ground truth EC measured by the soil sensor. Salinity level: S3>S2>S1.

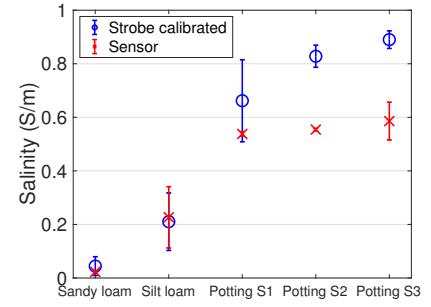

Figure 12: Salinity estimated by soil sensor and calibrated Strobe. At high salinity levels, Strobe is more accurate than the soil sensor.

Accuracy of soil sensor. Before comparing Strobe against the soil sensor, we need to understand the accuracy of the soil sensor itself. Previous studies [24, 30] show that soil sensors' permittivity estimation could suffer at high salinity but they can provide accurate EC reading, which matches the discussion in Section 3.6. To test whether this behavior also exists in the sensor we use, we setup two potting soil boxes with two salinity levels. We add the same amount of water into the two boxes to create 9 different moisture levels. We compute the ground truth moisture from the ratio of water volume to soil volume. Figure 10 compares the performance of the two boxes. Since we add the same amount of water into the two boxes at each moisture level, the soil sensor is supposed to report the same moisture value for the two boxes. However, it is obvious that the increase of salinity would increase measured moisture value, which means moisture measured by the sensor is inaccurate under high salinity levels.

Strobe's accuracy of soil moisture detection. All curves in Figure 11(a) present an increase of permittivity as moisture increases, indicating that Strobe can correctly detect soil moisture change in all three soil types under different salinity levels. We compare the moisture estimated by Strobe against that by the soil sensor in Figure 11(b). For the three potting soil boxes, since we add water to each box until soil is saturated, the boxes are supposed to have the same saturated soil moisture in the end, which is around $0.5 \mathrm{~m}^{3} / \mathrm{m}^{3}$ based on our measurements with the volumetric method. Strobe reports moisture at saturation with errors less than $0.03 \mathrm{~m}^{3} / \mathrm{m}^{3}$. In contrast, the soil sensor overestimates moisture for all potting soil boxes, similar to the results shown in Figure 10. The sensor has a maximum error of $0.1 \mathrm{~m}^{3} / \mathrm{m}^{3}$ as shown in Figure 11(b). For the other two soil types both having low EC, we treat moisture measured by the soil sensor as the ground truth. We observe a good match in silt loam and a deviation in sandy loam between soil sensor and Strobe. A possible reason for the deviation is that the real part of permittivity in sandy loam changes over frequency [31]. However, more experiments are needed to validate it.

Strobe's accuracy of soil salinity detection. Here we evaluate Strobe's capability of detecting different salinity levels. In Figure 11(a), we observe that the curves of Strobe are clearly separated while the curves of the soil sensor are overlapped at high salinity, which is caused by the sensor's erroneous permittivity reading. This means Strobe outperforms the soil sensor to tell different salinity levels.

However, as shown in Figure 11(c), Strobe always produces higher EC than the soil sensor since it operate at a high frequency, which is consistent with the discussion in Section 3.6. Here we treat the sensor results as the ground truth and apply Eq. 17 to calibrate Strobe. After the calibration, we observe a maximum EC deviation of $0.026 \mathrm{~S} / \mathrm{m}$.

Then we convert apparent EC to salinity, i.e., saturation extract EC, to present more intuitive results, as shown in Figure 12 . We only plot calibrated data for Strobe since its raw EC would cause significant overestimation of salinity. For each data point, we average the results for each soil box over salinity values estimated from the 3 highest moisture levels. We discard data of lower moisture levels because the method we use to compute salinity is imprecise for dryer soils [16]. Figure 12 shows that the soil sensor is not able to tell the salinity difference of the three potting soil boxes while Strobe can correctly distinguish all the salinity levels.

Comparing WARP with Atheros Wi-Fi card. Although the capability of commodity Wi-Fi cards to give accurate relative phase information has been proved by the rich 
Table 1: Comparison of WARP and Wi-Fi card results.

\begin{tabular}{c|c|c|c|c|c|c} 
& \multicolumn{2}{|c|}{ Permittivity } & \multicolumn{2}{c|}{ EC (S/m) } & Corr & Corr \\
& WARP & Wi-Fi & WARP & Wi-Fi & (phase) & (power) \\
\hline Test 1 & 6.59 & 6.59 & 0.15 & 0.18 & 0.9597 & 0.9971 \\
\hline Test 2 & 8.80 & 9.00 & 0.26 & 0.21 & 0.9981 & 0.9652 \\
\hline Test 3 & 14.95 & 14.95 & 0.41 & 0.48 & 0.9972 & 0.9226
\end{tabular}

AoA-related studies, such as SpotFi [18], we still need to verify that they work in soil. To do this, we conduct experiments with both WARP boards and Atheros Wi-Fi cards and use WARP results as a reference. When switching the data transmit and record devices from WARP boards to Wi-Fi cards, we keep transmit and receive antennas at the same locations. The Atheros Wi-Fi cards do not report reliable amplitudes, so we instead use RSSIs reported for each channel to estimate EC. Table 1 shows results of three tests with different moisture levels. WARP and Wi-Fi card report very similar apparent permittvity and EC results. We also compute the correlations between WARP results and Wi-Fi card results. As shown in Table 1, both phase and receive power have good correlations. Figure 13 shows the detailed data points in test 2 at the center frequencies of $2.4 \mathrm{GHz}$ channels. To get a clearer comparison, all RSSIs from the Wi-Fi card are subtracted by a constant in the figure. Overall, all the results of WARP and Wi-Fi card correlate well and the small deviations do not lead to significant deviations of permittivity and EC results shown in Table 1.

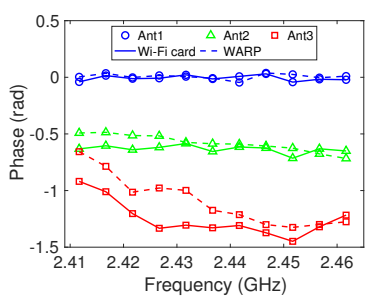

(a)

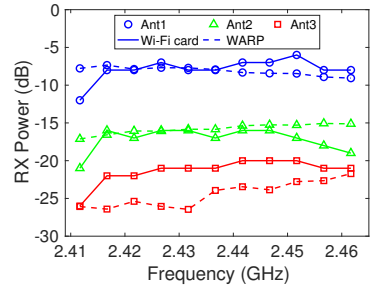

(b)
Figure 13: WARP and Wi-Fi card report similar phase and receive power.

\section{RELATED WORK}

While RF-based soil sensing has been well studied, our work is the first that makes it possible to use off-the-shelf low-cost Wi-Fi devices for detecting soil properties. We discuss related work in four main categories.

Soil sensing using RF. RF sensing techniques can be classified into two types. (i) Remote sensing techniques [3234] use the dependency of soil reflectivity on soil moisture to sense soil moisture. These approaches have low spatial resolution from $1 \mathrm{~m}$ to $10 \mathrm{~s}$ of $\mathrm{km}$, and can only detect soil moisture on shallow soil surface with a depth of a few centimeters. (ii) ToF-based techniques such as GPR [35] and TDR [36] provide good spatial resolution. However, these approaches rely on specialized ultra-wideband systems to get accurate ToF estimation, thus are very expensive. A recent study, LiquID[37], shows that lower cost UWB chips can be used for liquid identification. However, such a system still requires a lot of calibrations and the whole system can potentially cost 100 s of dollars.

Underground wireless sensor networks. The underground sensor networks [38-40] typically consist of underground soil probes and wireless communication nodes, where RF is responsible for communication, not sensing. The soil probes are usually commercially available soil sensors, whose high cost limits the scales of sensor networks. Using low-cost soil probes, however, is not ideal considering the loss of accuracy and capability. Most low-cost sensors, e.g., a capacitive sensor, can only sense moisture, not EC. A few studies [41-44] seek to reduce the cost by designing new moisture/EC-sensitive probes that can work with low-cost communication nodes, e.g., RFID or backscatter. However, designing a specialized probe could potentially increase cost.

AoA and ToF estimation on Wi-Fi devices. We build Strobe on existing AoA and ToF estimation technologies developed for commodity Wi-Fi devices [18, 19, 28, 45-47]. However, these technologies do not work for wave propagation in soil due to different reasons. It is unlikely to achieve the sub-nanosecond accuracy of Chronos [45] in soil due to the high attenuation of $5 \mathrm{GHz}$ signals. SpotFi's [45] accuracy benefits from $40 \mathrm{MHz}$ bandwidth and the carrier frequency of $5 \mathrm{GHz}$. To combat signal attenuation in soil, we instead use $20 \mathrm{MHz}$ channels at $2.4 \mathrm{GHz}$. To deal with multipath and amplitude variations due to impedance change or soil heterogeneity, we spliced all $2.4 \mathrm{GHz}$ channels. Existing work on channel splicing, however, only works for a single antenna [28, 47]. Our observations about hardware eliminates the exhaustive search for both PLL phase offset calibration [20] and channel splicing [28, 47] in prior work.

Other low-cost techniques. Besides ultra-wideband systems and Wi-Fi devices, there are some other commercially available RF devices that can provide ToF estimation, such as global positioning system (GPS) receivers [48]. GPS relies on ToF between satellites and the receiver for localization. However, its ToF resolution and penetration depth limit its use in ToF-based soil moisture sensing. Ranging techniques using ultrasound $[49,50]$ have been well studied for over-the-air wave propagation. However, ultrasound is not appropriate for ToF-based soil moisture estimation since it does not correlate very with moisture, which limits its applications of soil sensing to rely on reflectivity[51, 52].

\section{DISCUSSION \& FUTURE WORK}

Strobe takes the first step in leveraging Wi-Fi communication for estimating soil properties. However, for it to achieve 
its true potential, where a farmer with any Wi-Fi enabled device can infer soil properties, we plan to take Strobe in the following directions.

Conducting extensive evaluations. In this paper, we have shown limited results due to the difficulty of setting up measurements. These results, as a very first step, have shown Strobe's large potential in achieving good performance with simple calibrations. However, more measurements under more conditions need to be performed to rigorously evaluate Strobe's performance, just like how the existing commercial soil sensors are tested.

Making calibrations easier. Strobe requires two kinds of calibrations. (i) Calibrating frequency-dependent soil properties measured at $2.4 \mathrm{GHz}$ (Section 3.6): Similar to existing agricultural sensor solutions, this calibration only needs to be performed once for each soil type. The calibration data can then be reused over time and under different weather conditions. In the future, we will collect calibration data for the major soil types that only have a limited number; so we do not expect an enormous effort. Additionally, we can possibly reuse some calibration data from existing soil studies. (ii) Calibrating the hardware platform: Similar to AoA applications, currently we use cables with known lengths to calibrate the relative phase among antennas. We are investigating methods to get this done at the factory so that the system can be much easier to deploy and maintain.

Integration with commercial Wi-Fi devices. The Intel and Qualcomm Atheros 11n chipsets have shown the feasibility of providing CSI information to the user level, and we are working with other chip vendors to expose these values. Strobe's transmitter side, e.g., a smartphone, is simpler, which only requires a single antenna, and doesn't need to expose CSI values. Furthermore, since $2.4 \mathrm{GHz}$ of the spectrum is available in nearly all countries, we expect Strobe to be universally usable.

Sensing deeper in soil. We have tested Strobe over the $2.4 \mathrm{GHz}$ spectrum with depths up to $30 \mathrm{~cm}$. To make it sense deeper, our key insight is to use beamforming to increase the SNR. The challenge is to change the direction of the beamformed signal based on the moisture level of soil. We are actively investigating solutions to this problem. An alternative is to use the TV white space spectrum that can sense soil at depths deeper than $1 \mathrm{~m}$, which is sufficient for most broadacre crops and for horticulture.

Non-intrusive Sensing. Strobe measures the soil properties across two antennas. If the antennas are placed further apart, Strobe can help image soil. Such a technique makes it possible to map roots of plants without destroying them, which is known to be a hard problem in agriculture. It is also possible to use Strobe to measure physical properties of soil, such as compaction or porosity.
Price and Battery Life. Existing commercial soil sensors cost 100 s to 1000 s of dollars, especially the industrial grade soil EC sensors, and the ones we are now actively working with in a large scale multi-year agricultural IoT project.

Currently Strobe costs 10 s of dollars. Multiple features of Strobe can help bring down the system's overall cost to be sub-10 dollars. (i) Strobe does not require a specialized reader. (ii) Strobe only needs a single-band $(2.4 \mathrm{GHz}) \mathrm{Wi}$-Fi device to communicate with the device in soil. (iii) For the device in soil, although it is recommended to use a chipset with 3 antennas, a 2-antenna radio can work as well. The price of a typical IoT board with a 2-antenna $2.4 \mathrm{GHz}$ Wi-Fi chipset, an onboard ARM processor and batteries is similar to a Vocore2, or C.H.I.P., both of which cost less than 10 dollars.

The biggest cost in the system now is batteries, since the AA batteries we propose to use need replacements. We are actively researching on methods to recharge the batteries. In addition, we can use the deep sleep mode of Wi-Fi chipset to make batteries last longer, e.g., 4 AA batteries can last over a year. The device in soil only needs to wake up when receiving packets from a close-by surveying device containing a BSSID stored in it. Else, it operates in deep sleep mode.

To further reduce cost and improve battery life, we are investigating the use of a Wi-Fi based backscatter system in soil instead of an active transmitter. Based on our discussion with chip vendors, we also expect the cost of the system to be lower when manufactured at a larger scale, e.g., 10s of thousands of devices.

\section{SUMMARY}

In this paper we present a new technique, called Strobe, for estimating soil moisture and EC using Wi-Fi signals. The system estimates these parameters by measuring the relative time of flight of Wi-Fi signals between multiple antennas, and the ratios of the amplitudes of the signals. We have implemented Strobe on two SDR platforms and Wi-Fi cards. Our results show that Strobe can accurately estimate soil moisture and EC at various moisture and salinity levels.

Our vision is to enable a future where any farmer can take their smartphone close to soil and learn more about it. By avoiding expensive sensors that cost more than 100 dollars each, Strobe reduces the price for soil sensing, thereby taking a big step in enabling the adoption of data-driven agriculture techniques by small holder farmers.

\section{ACKNOWLEDGMENTS}

We sincerely thank our shepherd, Domenico Giustiniano, and the anonymous reviewers for their valuable feedback. We thank Colleen Josephson, Deepak Vasisht, and Manikanta Kotaru for their constructive input and help. This research was done at Microsoft. Jian Ding was supported in part by NSF Award \#1518916. 


\section{REFERENCES}

[1] Soil electrical conductivity, soil quality kit - guide for educators, usda nrcs. https://www.agric.wa.gov.au/horticulture/soil-moisturemonitoring-selection-guide.

[2] Soil moisture monitoring: a selection guide, department of primary industries and regional development, government of australia, 5th sep, 2018. https://www.agric.wa.gov.au/horticulture/soil-moisturemonitoring-selection-guide.

[3] Carlos MP Vaz, Scott Jones, Mercer Meding, and Markus Tuller. Evaluation of standard calibration functions for eight electromagnetic soil moisture sensors. Vadose Zone Journal, 12(2), 2013.

[4] G Kargas and P Kerkides. Evaluation of a dielectric sensor for measurement of soil-water electrical conductivity. Fournal of Irrigation and Drainage Engineering, 136(8):553-558, 2010.

[5] Deepak Vasisht, Zerina Kapetanovic, Jongho Won, Xinxin Jin, Ranveer Chandra, Sudipta N. Sinha, Ashish Kapoor, Madhusudhan Sudarshan, and Sean Stratman. FarmBeats: An IoT platform for data-driven agriculture. In Proceedings of the 14th USENIX Symposium on Networked Systems Design and Implementation (NSDI'17), pages 515-529, 2017.

[6] Milton Whitney et al. Instructions for taking samples of soil for moisture determinations. 1894.

[7] EA Colman. The place of electrical soil-moisture meters in hydrologic research. Eos, Transactions American Geophysical Union, 27(6):847-853, 1946.

[8] Harrison E Patten. Heat transference in soils. 1909.

[9] LA Richards. Soil moisture tensiometer materials and construction. Soil Sci, 53(4):241-248, 1942

[10] Wilford Gardner and Don Kirkham. Determination of soil moisture by neutron scattering. Soil Science, 73(5):391-402, 1952.

[11] G Clarke Topp, JL Davis, and Aa P Annan. Electromagnetic determination of soil water content: Measurements in coaxial transmission lines. Water resources research, 16(3):574-582, 1980.

[12] Kurt Roth, Rainer Schulin, Hannes Flühler, and Werner Attinger. Calibration of time domain reflectometry for water content measurement using a composite dielectric approach. Water Resources Research, 26(10):2267-2273, 1990.

[13] John O Curtis. Moisture effects on the dielectric properties of soils. IEEE transactions on geoscience and remote sensing, 39(1):125-128, 2001.

[14] MA Hilhorst. A pore water conductivity sensor. Soil Science Society of America fournal, 64(6):1922-1925, 2000.

[15] Harry M Jol. Ground penetrating radar theory and applications. elsevier, 2008.

[16] Decagon Devices. GS3 water content, ec temperature sensor: OperatorâĂŹs manual. Pullman: Decagon Devices, 2016.

[17] DA Robinson, Scott B Jones, JM Wraith, Daniel Or, and SP Friedman. A review of advances in dielectric and electrical conductivity measurement in soils using time domain reflectometry. Vadose Zone fournal, 2(4):444-475, 2003.

[18] Manikanta Kotaru, Kiran Joshi, Dinesh Bharadia, and Sachin Katti. SpotFi: Decimeter level localization using WiFi. In Proceedings of the 2015 ACM SIGCOMM Conference, volume 45, pages 269-282. ACM, 2015.

[19] Jie Xiong and Kyle Jamieson. ArrayTrack: A fine-grained indoor location system. In Proceedings of the 10th USENIX Symposium on Networked Systems Design and Implementation (NSDI'13), pages 71-84, Lombard, IL, 2013. USENIX.

[20] Jon Gjengset, Jie Xiong, Graeme McPhillips, and Kyle Jamieson. Phaser: Enabling phased array signal processing on commodity WiFi access points. In Proceedings of the 20th annual international conference on Mobile computing and networking (MobiCom'14), pages 153-164. ACM, 2014.
[21] Yaxiong Xie, Yanbo Zhang, Jansen Christian Liando, and Mo Li. SWAN: Stitched Wi-Fi antennas. In Proceedings of the 24th Annual International Conference on Mobile Computing and Networking (MobiCom'18), pages 51-66. ACM, 2018.

[22] ZHI Sun, Ian F Akyildiz, and Gerhard P Hancke. Dynamic connectivity in wireless underground sensor networks. IEEE Transactions on Wireless Communications, 10(12):4334-4344, 2011.

[23] Zhe Chen, Zhongmin Li, Xu Zhang, Guorong Zhu, Yuedong Xu, Jie Xiong, and Xin Wang. AWL: Turning spatial aliasing from foe to friend for accurate WiFi localization. In Proceedings of the 13th International Conference on Emerging Networking EXperiments and Technologies (CoNEXT '17), pages 238-250, New York, NY, USA, 2017. ACM.

[24] Ullrich Dettmann and Michel Bechtold. Evaluating commercial moisture probes in reference solutions covering mineral to peat soil conditions. Vadose Zone fournal, 17(1), 2018.

[25] Svatopluk Matula, Kamila Bát'ková, and Wossenu Legese. Laboratory performance of five selected soil moisture sensors applying factory and own calibration equations for two soil media of different bulk density and salinity levels. Sensors, 16(11):1912, 2016.

[26] GC Topp, M Yanuka, WD Zebchuk, and S Zegelin. Determination of electrical conductivity using time domain reflectometry: Soil and water experiments in coaxial lines. Water Resources Research, 24(7):945-952, 1988.

[27] Daniel Halperin, Wenjun Hu, Anmol Sheth, and David Wetherall. Tool release: Gathering $802.11 \mathrm{n}$ traces with channel state information. SIGCOMM Comput. Commun. Rev., 41(1):53-53, January 2011.

[28] Yaxiong Xie, Zhenjiang Li, and Mo Li. Precise power delay profiling with commodity WiFi. In Proceedings of the 21st Annual International Conference on Mobile Computing and Networking (MobiCom'15), pages 53-64. ACM, 2015.

[29] Rice University. WARP project. http://warpproject.org.

[30] SJ Lim, MN Shin, JK Son, JD Song, KH Cho, SH Lee, JH Ryu, and JY Cho. Evaluation of soil pore-water salinity using a decagon gs3 sensor in saline-alkali reclaimed tidal lands. Computers and Electronics in Agriculture, 132:49-55, 2017.

[31] Myron C Dobson, Fawwaz T Ulaby, Martti T Hallikainen, and Mohamed A El-Rayes. Microwave dielectric behavior of wet soil-part II: Dielectric mixing models. IEEE Transactions on Geoscience and Remote Sensing, (1):35-46, 1985.

[32] JR Wang and BJ Choudhury. Remote sensing of soil moisture content, over bare field at $1.4 \mathrm{ghz}$ frequency. Journal of Geophysical Research: Oceans, 86(C6):5277-5282, 1981.

[33] Thomas J Jackson. III. measuring surface soil moisture using passive microwave remote sensing. Hydrological processes, 7(2):139-152, 1993.

[34] Binayak P Mohanty, Michael H Cosh, Venkat Lakshmi, and Carsten Montzka. Soil moisture remote sensing: State-of-the-science. Vadose Zone fournal, 16(1), 2017.

[35] JA Huisman, SS Hubbard, JD Redman, and AP Annan. Measuring soil water content with ground penetrating radar. Vadose zone journal, 2(4):476-491, 2003

[36] K Noborio. Measurement of soil water content and electrical conductivity by time domain reflectometry: a review. Computers and electronics in agriculture, 31(3):213-237, 2001.

[37] Ashutosh Dhekne, Mahanth Gowda, Yixuan Zhao, Haitham Hassanieh, and Romit Roy Choudhury. Liquid: A wireless liquid identifier. In Proceedings of the 16th Annual International Conference on Mobile Systems, Applications, and Services (MobiSys'18), pages 442-454. ACM, 2018.

[38] M Can Vuran and Agnelo R Silva. Communication through soil in wireless underground sensor networks-theory and practice. In Sensor Networks, pages 309-347. Springer, 2010. 
[39] Yiwei Zhuo, Hongzi Zhu, Hua Xue, and Shan Chang. Perceiving accurate CSI phases with commodity WiFi devices. In INFOCOM 2017IEEE Conference on Computer Communications, IEEE, pages 1-9. IEEE, 2017.

[40] Mahta Moghaddam, Dara Entekhabi, Yuriy Goykhman, Ke Li, Mingyan Liu, Aditya Mahajan, Ashutosh Nayyar, David Shuman, and Demosthenis Teneketzis. A wireless soil moisture smart sensor web using physics-based optimal control: Concept and initial demonstrations. IEEE Journal of Selected Topics in Applied Earth Observations and Remote Sensing, 3(4):522-535, 2010.

[41] Azhar Hasan, Rahul Bhattacharyya, and Sanjay Sarma. A monopolecoupled rfid sensor for pervasive soil moisture monitoring. In Antennas and Propagation Society International Symposium (APSURSI), 2013 IEEE, pages 2309-2310. IEEE, 2013.

[42] Shuvashis Dey, Nemai Karmakar, Rahul Bhattacharyya, and Sanjay Sarma. Electromagnetic characterization of soil moisture and salinity for UHF RFID applications in precision agriculture. In Microwave Conference (EuMC), 2016 46th European, pages 616-619. IEEE, 2016.

[43] Spyridon-Nektarios Daskalakis, Stylianos D Assimonis, Eleftherios Kampianakis, and Aggelos Bletsas. Soil moisture scatter radio networking with low power. IEEE Transactions on Microwave Theory and Techniques, 64(7):2338-2346, 2016.

[44] Md Mazidul Islam, Kimmo Rasilainen, and Ville Viikari. Implementation of sensor rfid: Carrying sensor information in the modulation frequency. IEEE Transactions on Microwave Theory and Techniques, 63(8):2672-2681, 2015.

[45] Deepak Vasisht, Swarun Kumar, and Dina Katabi. Decimeter-level localization with a single WiFi access point. In Proceedings of the 13th
USENIX Symposium on Networked Systems Design and Implementation (NSDI'16), pages 165-178, 2016.

[46] Swarun Kumar, Stephanie Gil, Dina Katabi, and Daniela Rus. Accurate indoor localization with zero start-up cost. In Proceedings of the 20th annual international conference on Mobile computing and networking (MobiCom'14), pages 483-494. ACM, 2014.

[47] Jie Xiong, Karthikeyan Sundaresan, and Kyle Jamieson. ToneTrack: Leveraging frequency-agile radios for time-based indoor wireless localization. In Proceedings of the 21st Annual International Conference on Mobile Computing and Networking (MobiCom '15), pages 537-549, New York, NY, USA, 2015. ACM.

[48] Elliott Kaplan and Christopher Hegarty. Understanding GPS: principles and applications. Artech house, 2005.

[49] Andy Ward, Alan Jones, and Andy Hopper. A new location technique for the active office. IEEE Personal communications, 4(5):42-47, 1997.

[50] Nissanka B. Priyantha, Anit Chakraborty, and Hari Balakrishnan. The cricket location-support system. In Proceedings of the 6th Annual International Conference on Mobile Computing and Networking (MobiCom '00), pages 32-43, New York, NY, USA, 2000. ACM.

[51] DA Robinson, CS Campbell, JW Hopmans, Brian K Hornbuckle, Scott B Jones, R Knight, F Ogden, J Selker, and O Wendroth. Soil moisture measurement for ecological and hydrological watershed-scale observatories: A review. Vadose Zone fournal, 7(1):358-389, 2008.

[52] Nobutaka Hiraoka, Takefumi Suda, Kazuhiro Hirai, Katsuhiko Tanaka, Kazunari Sako, Ryoichi Fukagawa, Makoto Shimamura, and Asako Togari. Improved measurement of soil moisture and groundwater level using ultrasonic waves. Japanese Journal of Applied Physics, 50(7S):07HC19, 2011. 